\title{
Pupillary Abnormalities in Congenital Neurosyphilis
}

\author{
M.B.M. Sundaram
}

\begin{abstract}
Pupils in congenital neurosyphilis differ from the classic Argyll-Robertson pupil in acquired cases, and often tend to be large and unreactive. Constriction to pilocarpine in the reported patient would suggest that peripheral parasympathetic damage is responsible for such pupillary findings. Congenital neurosyphilis should be included among the causes of "tonic" pupil.
\end{abstract}

RÉSUMÉ: Anomalies pupillaires dans la neurosyphilis congénitale Les pupilles dans la neurosyphilis congénitale diffèrent de la pupille Argyl-Robertson classique des cas acquis: elles ont tendance à être grandes et non réactives. Le fait que la pupille du patient ici rapporté pouvait se contracter après pilocarpine suggère qu'une atteinte parasympathique périphérique est probablement responsable des changements. Il faut donc inclure la syphilis congénitale parmi les causes d'une pupille "tonique".

Can. J. Neurol. Sci. 1985: 12:134-135

The classic, miotic, light-near dissociated Argyll-Robertson (AR) pupil of neurosyphilis is well known. However, the occurrence of large, unreactive pupils in congenital neurosyphilis is not widely recognized. Such a case is presented with pharmacological evidence that the responsible lesion in these congenital cases is likely peripheral at or distal to the ciliary ganglion.

\section{CASE REPORT}

This 53 year old patient was admitted with phenytoin overdose. She had a well documented history of congenital syphilis. At the age of 9 years, her school performance began to deteriorate. Serological examination then (1945) showed strongly positive blood and CSF Wasserman reaction. CSF colloidal gold test showed a paretic curve. She was treated first with bismuth, malaria fever therapy and subsequently penicillin. Although mental deterioration was arrested, the patient was left with low average intelligence and immature personality. The hospital records also documented long standing large unreactive pupils, mild ataxia of gait with proprioceptive impairment in feet, absent tendon reflexes and occasional urinary incontinence. None of these, however, progressed. At the age of 41 years, she was placed on phenytoin following several tonic-clonic seizures.

Examination during the present admission revealed large pupils in both normal room illumination and darkness; the right was $8 \mathrm{~mm}$ and the left $7 \mathrm{~mm}$ in diameter (Figure 1A). The left was slightly oval. Pupillary margins were slightly irregular. There was no direct or consensual response to light in either eye. Slip lamp examination did not show any segmental reactivity. There was no constriction to near stimulus maintained for 10 minutes. Corneas were clear. Ophthalmoscopy showed peripheral pigmentary disruption in both eyes. There was mild bilateral optic atrophy. Corrected distance visual acuity was $20 / 40$ bilaterally. There was exotropia with full ocular movements.

Investigations showed positive serum FTA-ABS (fluorescent treponemal antibody absorption) and reactive RST (Reagin screening test). Serum VDRL was negative. CSF was normal with negative RST. These results indicated past infection with syphilis. CT scan of the brain showed several small round areas of calcification in the frontal and basal ganglia regions; although nonspecific, these were felt to be consistent with calcified cerebral gumma. Toxoplasmosis serology was negative. Pattern reversal visual evoked responses were normal.

$0.1 \%$ pilocarpine drops (prepared by diluting $0.1 \mathrm{cc}$ of $1 \%$ pilocarpine with $0.9 \mathrm{cc}$ of normal saline in a tuberculin syringe and found to have no effect on the pupils in normal volunteers) were placed into either eye. Both pupils constricted to about $3 \mathrm{~mm}$ in diameter after 20 minutes (Figure 1B). The photographs were taken in constant room illumination with the patient maintaining distant fixation. Activities that require near fixation like reading, were avoided between the two sets of photographs.

\section{Discussion}

Although Merritt et al. (1946) mention, in their book on neurosyphilis, that pupils in congenital paretic and tabetic cases are "usually dilated rather than miotic", standard neurology text books make no reference or only a fleeting comment to such pupillary findings. The exact frequency of this pupillary finding among patients with congenital neurosyphilis is not known. Merritt et al. (1946) found large and "stiff"' pupils in 3 of their 23 patients with congenital paretic neurosyphilis; they mention unequal and stiff pupils in 6 other cases, but do not specify pupillary size. These authors also described two patients 


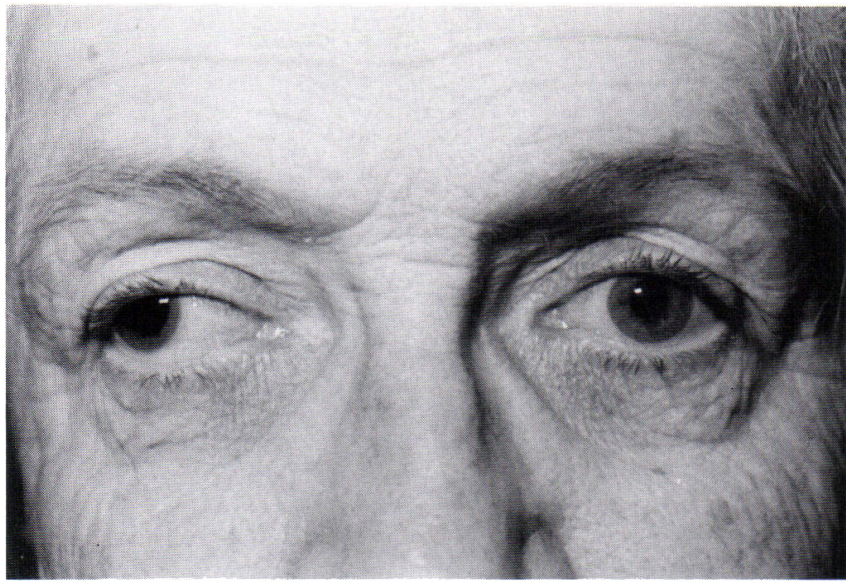

Figure $1 A-$ Large pupils before pilocarpine.

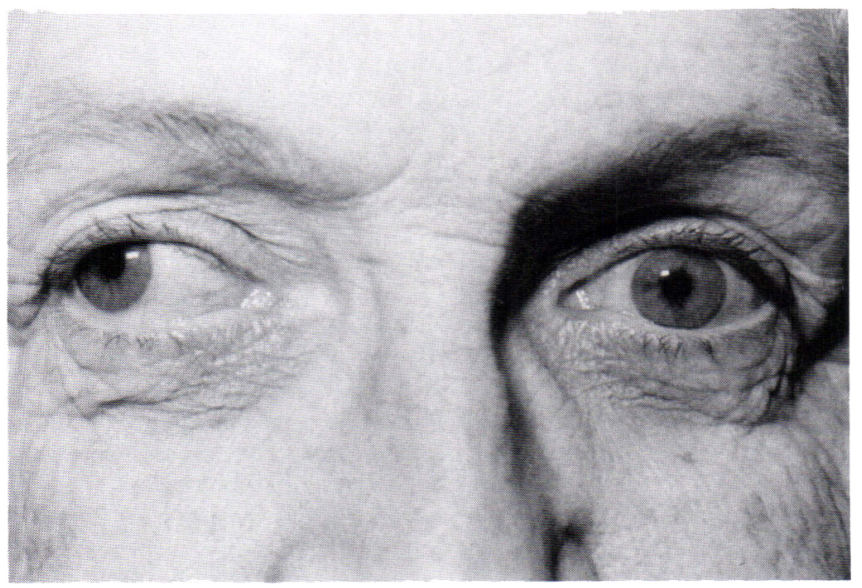

Figure $1 B-$ Pupillary constriction following $0.1 \%$ pilocarpine.

with congenital tabetic neurosyphilis, one with large, unreactive pupils and the other with unequal, fixed pupils. Cases of neurosyphilis continue to occur and the incidence might be as high as about 5000 new cases in United States per year (Hotson, 1981); hence there should be an increased awareness of the pupillary changes in congenital cases.

Is the large unreactive pupil of congenital syphilis a variant of the classic Argyll-Robertson pupil? In a detailed review of AR pupil, Loewenfeld (1969) has shown that AR pupil, in addition to showing light-near dissociation, is by definition miotic. She and Lowenstein (1956) have hypothesized that the responsible lesion should be in the midbrain, rostro-dorsal to the oculomotor nucleus. Miosis was thought to result from damage to the supranuclear inhibitory fibers in that region. These observations would indicate that the pathogenesis and site of pathology of the large pupil in congenital syphilis are different from the classic AR pupil.
The irregularly dilated non-reactive pupils in the reported patient with constriction to $0.1 \%$ pilocarpine suggest "tonic" pupils. The absence of pupillary constriction following sustained near vision in this patient is somewhat unusual but can rarely be a feature of "tonic" pupil (Loewenfeld, I.E., Thompson, H.S., 1967). Idiopathic tonic pupil is often unilateral, although this eventually could become bilateral. 104 of 150 patients with tonic pupils, reported by Thompson (1971), had unilateral changes. In that series, 7 of 21 patients with bilateral, "neuropathic tonic pupils" had reactive VDRL and FTA-ABS. Postganglionic parasympathetic denervation with simultaneous reinervation of fibres to intraocular muscles is considered responsible for tonic pupil (Thompson, 1971). The resulting denervation supersensitivity of iris sphinctor is best confirmed by constriction of pupil to dilute pilocarpine, as was shown in patient reported. There is pathologic evidence of neuronal degeneration in ciliary ganglion in patients with tonic pupil (Harriman and Garland, 1968; Selhorst et al., 1984; Ulrich, 1980). Hence, it seems likely that peripheral parasympathetic denervating lesion at or distal to the ciliary ganglion is probably responsible for the large irregular pupil in congenital neurosyphilis; the lack of reinervation in these patients perhaps explains the complete unreactivity to light even on slit lamp examination. However, this would remain speculative until pathologic confirmation is available.

It should be emphasized that pupillary changes and other neurological deficits in congenital neurosyphilis (as in acquired cases) can persist indefinitely after successful therapy and do not necessarily indicate active disease.

\section{ACKNOWLEDGEMENT}

I would like to thank Dr. Ken Romanchuk for his comments and photographs.

\section{REFERENCES}

Harriman DGF, Garland H (1968) The Pathology of Adie's Syndrome. Brain. 91: 401-418.

Hotson JR (1981) Modern Neurosyphilis: A partially treated chronic meningitis. Western J. of Med. 135: 191-200.

Loewenfeld IE (1969) The Argyll-Robertson pupil, 1869-1969: A critical survey of the literature. Survey of ophthalmology. 14: 199-299.

Loewenfeld IE, Thompson HS (1967) The tonic pupil: A re-evaluation. Am. J. of Ophth. 63: 46-87.

Lowenstein O (1956). The Argyll-Robertson pupillary syndrome. Am. J. of Ophth. 42: 105-121.

Merritt HH, Adam RD, Soloman HC (1946) Neurosyphilis. Chap.9, Congenital neurosypilis, pp. 289-319. Oxford University Press, New York.

Selhorst JB, Madge G, Ghatak NR (1984) The neuropathology of the Holmes-Adie Syndrome (Abstracted). Ann. of Neurol. 16: 138.

Thompson HS (1971) Adie's syndrome: some new observations. Trans. Am. Ophth. Society. 75: 587-626.

Ulrich J (1980) Morphological basis of Adie's syndrome. Eur. Neurol. 19: 390-395. 